\title{
Myeloproliferative neoplasm-driving Calr frameshift promotes the development of pulmonary hypertension in mice
}

\author{
Keiji Minakawa ${ }^{1 \dagger}$, Tetsuro Yokokawa ${ }^{2 \dagger}$, Koki Ueda$^{1}$, Osamu Nakajima ${ }^{3}$, Tomofumi Misaka ${ }^{2}$, Yusuke Kimishima ${ }^{2}$ \\ Kento Wada ${ }^{2}$, Yusuke Tomita², Saori Miura' ${ }^{1}$ Yuka Sato ${ }^{1}$, Kosaku Mimura' ${ }^{1}$, Koichi Sugimoto ${ }^{2,4}$, \\ Kazuhiko Nakazato ${ }^{2}$, Kenneth E. Nollet ${ }^{1}$, Kazuei Ogawa ${ }^{5}$, Takayuki Ikezoe ${ }^{5}$, Yuko Hashimoto ${ }^{6}$, \\ Yasuchika Takeishi ${ }^{2}$ and Kazuhiko Ikeda ${ }^{1 *}$ (D)
}

\begin{abstract}
Frameshifts in the Calreticulin (CALR) exon 9 provide a recurrent driver mutation of essential thrombocythemia (ET) and primary myelofibrosis among myeloproliferative neoplasms (MPNs). Here, we generated knock-in mice with murine Calr exon 9 mimicking the human CALR mutations, using the CRISPR-Cas9 method. Knock-in mice with del10 [Caldel10/WT (wild-type) mice] exhibited an ET phenotype with increases of peripheral blood (PB) platelets and leukocytes, and accumulation of megakaryocytes in bone marrow (BM), while those with ins2 (Calrins2 ${ }^{\text {IWT }}$ mice) showed a slight splenic enlargement. Phosphorylated STAT3 (pSTAT3) was upregulated in BM cells of both knock-in mice. In BM transplantation (BMT) recipients from Calrdel10/WT mice, although PB cell counts were not different from those in BMT recipients from Calr ${ }^{\text {WT }}$ WT mice, Calr ${ }^{\text {del10NWT }}$ BM-derived macrophages exhibited elevations of PSTAT3 and Endothelin-1 levels. Strikingly, BMT recipients from Calr del10NT mice developed more severe pulmonary hypertension (PH) — which often arises as a comorbidity in patients with MPNs - than BMT recipients from Calr ${ }^{\text {WT/NT }}$ mice, with pulmonary arterial remodeling accompanied by an accumulation of donor-derived macrophages in response to chronic hypoxia. In conclusion, our murine model with the frameshifted murine Calr presented an ET phenotype analogous to human MPNs in molecular mechanisms and cardiovascular complications such as PH.
\end{abstract}

Keywords: CALR, Pulmonary hypertension, Myeloproliferative neoplasms, Macrophage, Essential thrombocythemia

\section{To the editor,}

CALR frameshifts provide a recurrent myeloproliferative neoplasm (MPN) driver [1]. Pulmonary hypertension $(\mathrm{PH})$ is a life-threatening cardiopulmonary disease characterized by increased pulmonary arterial (PA) pressure. Bone marrow (BM)-derived cells and perivascular

${ }^{*}$ Correspondence: kazu-ike@fmu.ac.jp

${ }^{\dagger}$ Keiji Minakawa and Tetsuro Yokokawa equally contributed to this work

1 Department of Blood Transfusion and Transplantation Immunology,

School of Medicine, Fukushima Medical University, 1 Hikarigaoka, Fukushima 960-1295, Japan

Full list of author information is available at the end of the article inflammatory infiltrates contribute to PA remodeling in $\mathrm{PH}[2,3]$. Among 5 etiological groups, the WHO group$\mathrm{V}$ PH encompasses multifactorial mechanisms, including MPNs, which are often complicated by $\mathrm{PH}$, with $5 \%-60 \%$ of the prevalence [4-6]. MPN-related PH is associated with crucial features, such as thromboembolism and hypermetabolic state [5]. However, the association of $\mathrm{PH}$ with CALR mutation remains uncertain. Here, we generated Calr ${ }^{\text {del10/WT }}$ and Calr ${ }^{\text {ins2/WT }}$ knock-in mice (Fig. 1a, Additional file 1: Fig. S1), investigated their hematopoiesis, and clarified the role of hematopoietic Calr mutation original author(s) and the source, provide a link to the Creative Commons licence, and indicate if changes were made. The images or other third party material in this article are included in the article's Creative Commons licence, unless indicated otherwise in a credit line to the material. If material is not included in the article's Creative Commons licence and your intended use is not permitted by statutory regulation or exceeds the permitted use, you will need to obtain permission directly from the copyright holder. To view a copy of this licence, visit http://creativecommons.org/licenses/by/4.0/. The Creative Commons Public Domain Dedication waiver (http://creativeco mmons.org/publicdomain/zero/1.0/) applies to the data made available in this article, unless otherwise stated in a credit line to the data. 
in $\mathrm{PH}$ using $\mathrm{BM}$ transplantation (BMT) and chronic hypoxia, which provokes $\mathrm{PH}$ [7].

In a public database [8], 138 CALR frameshifts, including the major del52 and ins5 [1], and another frameshift in 2 MPN patients (ET, myelofibrosis) exactly matching the Calr-del10, have been noted in patients with hematopoietic cancers, mostly MPNs (Additional file 7: Table S1, Additional file 1: Fig. S1f). Mouse models carrying frameshifted CALR showed ET or, rarely, myelofibrosis [9]. Likewise, Calr ${ }^{\text {del10/WT }}$ mice developed ET with phosphorylated STAT3 (pSTAT3) and cell-surface thrombopoietin-receptor (TpoR) expressions suggested to accompany mutant CALR [10], whereas Calr ${ }^{\text {ins2/WT }}$ mice showed a slight splenic enlargement (Fig. 1b, Additional files 2, 3: Fig. S2-S3).

To elucidate the roles of hematopoietic Calr mutation in $\mathrm{PH}$, we performed non-competitive BMT from Cal$r^{\text {del10/WT }}$ mice (Fig. 1c), as we reconstituted Jak2V617F $\mathrm{F}^{+}$ MPNs [11]. At 4 weeks after BMT, the engraftments were achieved in the BMT recipients from Calr ${ }^{\text {del10/WT }}$ mice (del-R, Fig. 1d), but their PB cell counts (Additional files 4: Fig. S4) and BM megakaryocytic distribution did not differ from BMT recipients from Calr ${ }^{\mathrm{WT} / \mathrm{WT}}$ mice (WT-R). We assessed right heart hemodynamics and right ventricular (RV) hypertrophy, showing that neither RV systolic pressure (RVSP) nor right ventricle/left ventricle-plusseptum weight ratio $(\mathrm{RV} / \mathrm{LV}+\mathrm{S})$ differed between WT-R and del-R. Subsequently, del-R were exposed to chronic hypoxia $\left(10 \% \mathrm{O}_{2}\right)$ for 3 weeks. Strikingly, although chronic hypoxia elevated RVSP and RV/LV + S in both WT-R and del- $R$, these levels in del- $R$ were significantly greater than in WT-R, suggesting that hematopoietic Calr mutation promotes $\mathrm{PH}$ (Fig. 1c-e).
Lung histology showed significant increases in PA medial wall thickness and muscularization, indicated by $\alpha$ smooth muscle actin, without thrombosis in del-R compared to WT-R under chronic hypoxia, whereas $\mathrm{F} 4 / 80^{+}$ macrophages rather than $\mathrm{TpoR}^{+}$cells were increased specifically in PA regions in both WT-R and del-R (Fig. 1fh, Additional file 3: Fig. S3e). However, pSTAT3 levels were elevated in the lungs of del-R compared to WT-R after chronic hypoxia. The expression of Endothelin-1, an important vasoactive peptide involving PA remodeling in $\mathrm{PH}[4,5]$, was also increased in the lungs of del- $\mathrm{R}$ compared to WT-R under chronic hypoxia (Fig. 2a, b, Additional file 5: Fig. S5). We visualized the Calr del10/WT BM-derived cells using CAG-EGFP: in the lungs of BMT

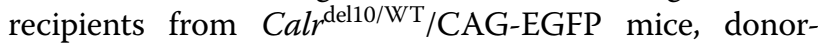
derived macrophages accumulated in PA regions, but donor-derived cells were not observed in vascular walls (Fig. 2c), suggesting that Calr del10/WT BM-derived macrophages migrated into the PA regions.

RNA sequencing of hematopoietic progenitors showed Calr-del10 activated JAK-STAT pathway, as well as cardiac-hypertrophy pathway that includes upregulation of Endothelin-1. Also, human CALR-del52 introduction upregulated Endothelin-1 in a macrophage cell line (Additional file 6: Fig. S6). We next obtained Calr ${ }^{\text {del10/WT }}$ macrophages by culturing BM-mononuclear cells (BM-MNCs) in the presence of M-CSF (Fig. 2d, e). The increases in the Endothelin-1 and pSTAT3 levels did not show the statistical difference between in Calr ${ }^{\mathrm{WT} / \mathrm{WT}}$ and $\mathrm{Calr}^{\mathrm{del10/ \textrm {WT }}}$ macrophages at baseline, but these levels in Calr del10/WT macrophages were significantly more upregulated compared to Calr ${ }^{\mathrm{WT} / \mathrm{WT}}$ macrophages after lipopolysaccharide stimulation (Fig. 2f, g). These data suggest that BM-derived

\footnotetext{
(See figure on next page.)
}

Fig. 1 Hematopoietic cells with Calr mutation exacerbate the development of pulmonary hypertension in response to chronic hypoxia. a The knock-in mice with C57BL/6 J background carrying frameshifted murine Calr, del10 (Calrdel10/WT mice) and ins2 (Calr ins2/WT mice) were generated using the CRISPR-Cas9 method. Structure of wild-type (WT) and frameshifted murine CALR proteins are shown. Both generated mutant proteins with shortened calcium-buffering sites and absent KDEL sequence, which is the signal to retain the CALR protein in the endoplasmic reticulum. b Leukocyte (white blood cell) counts (WBC), red blood cell counts (RBC), and platelet counts (PLT) in WT mice (Calr ${ }^{\text {WT/WT }}$ mice, $\left.n=21\right)$, Calrins2/WT mice $(n=17)$, and Calrdel10NT mice $(n=16)$ in the peripheral blood. ${ }^{*} P<0.05$ versus the WT group. $\mathbf{c}$ Schematic diagram of the experimental design of bone marrow (BM) transplantation (BMT). BM cells from control Calr ${ }^{\text {WT } N T}$ mice or Calr del10/WT mice were injected into the lethally irradiated WT mice (C57BL/6 J mice). Four weeks after BMT, the recipient mice transplanted with the BM cells from the Calr ${ }^{\text {WT }}$ WT mice (WT-R) or Calr ${ }^{\text {dello/WT }}$ mice (del-R) were subjected to normoxia $\left(21 \% \mathrm{O}_{2}\right)$ or chronic hypoxia $\left(10 \% \mathrm{O}_{2}\right)$ for 3 weeks. $\mathbf{d}$ Allele frequency of the mutant Calr in the peripheral leukocytes of recipient mice at 4 weeks after BMT ( $n=15$, each). e Right ventricular (RV) systolic pressure (RVSP) and RV hypertrophy determined by dividing the RV weight by the left ventricular weight including the septum (RV/LV $+S)(n=6-8)$. f Representative hematoxylin-eosin (HE) staining and immunohistochemistry with antibodies to anti-a smooth muscle actin (aSMA) and anti-F4/80 images in the lung of BMT recipient mice from Calr ${ }^{\text {WT }}{ }^{\text {WT }}$ or Cald del10/WT mice. Scale bars, $50 \mu \mathrm{m}$. $\mathbf{g}$ Quantitative analysis of the percentage of muscularized distal pulmonary arteries in aSMA-immunostained sections ( $n=3$, each). $\mathbf{h}$ Quantitative analysis of the pulmonary perivascular macrophages determined as F4/80-positive cells, per 30 vessels $\left(n=5\right.$, each). Data are presented as means \pm SEM. $\mathbf{d}, \mathbf{e}, \mathbf{g}, \mathbf{h}^{*} P<0.05$ versus the corresponding normoxia group and ${ }^{\dagger} P<0.05$ versus the corresponding BMT recipient mice from Calr ${ }^{\text {WT } / W T}$ mice. WT-R, recipient mice transplanted with BM cells from Calr ${ }^{\text {WT } / W T}$ mice; del-R, recipient mice transplanted with BM cells from Caldel10/WT mice. Oligonucleotides and antibodies used are listed in Additional files 8,9 


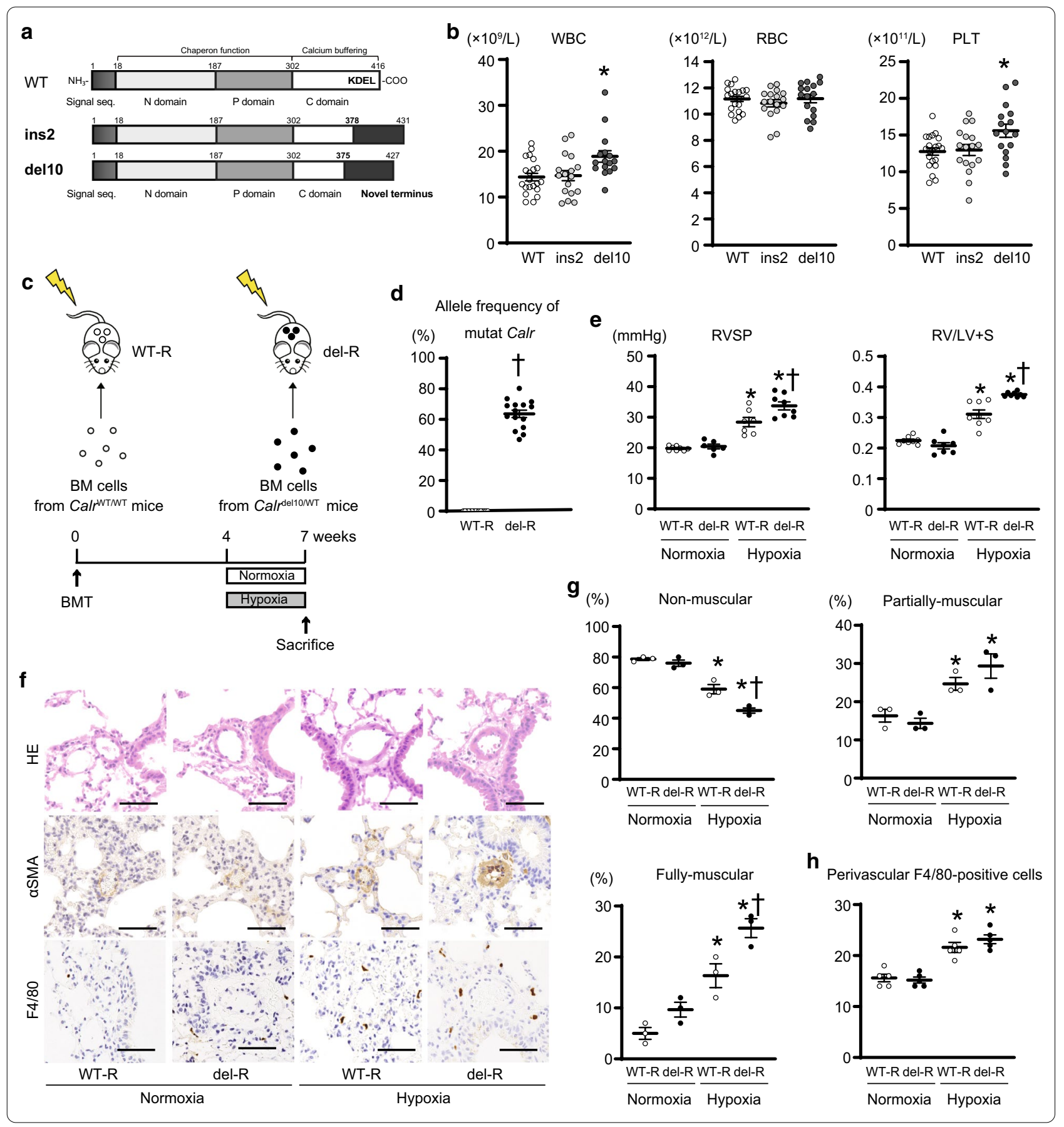

macrophages with the Calr mutation played important roles in the PA remodeling.

To date, most of studies for $\mathrm{PH}$ in MPN patients lack information about driver mutations, although a retrospective study indicated higher prevalence of CALR mutations in ET patients with PH than those without [6]. Besides megakaryocyte lineage with TpoR expression, a recent study indicated that transcriptional misregulation 


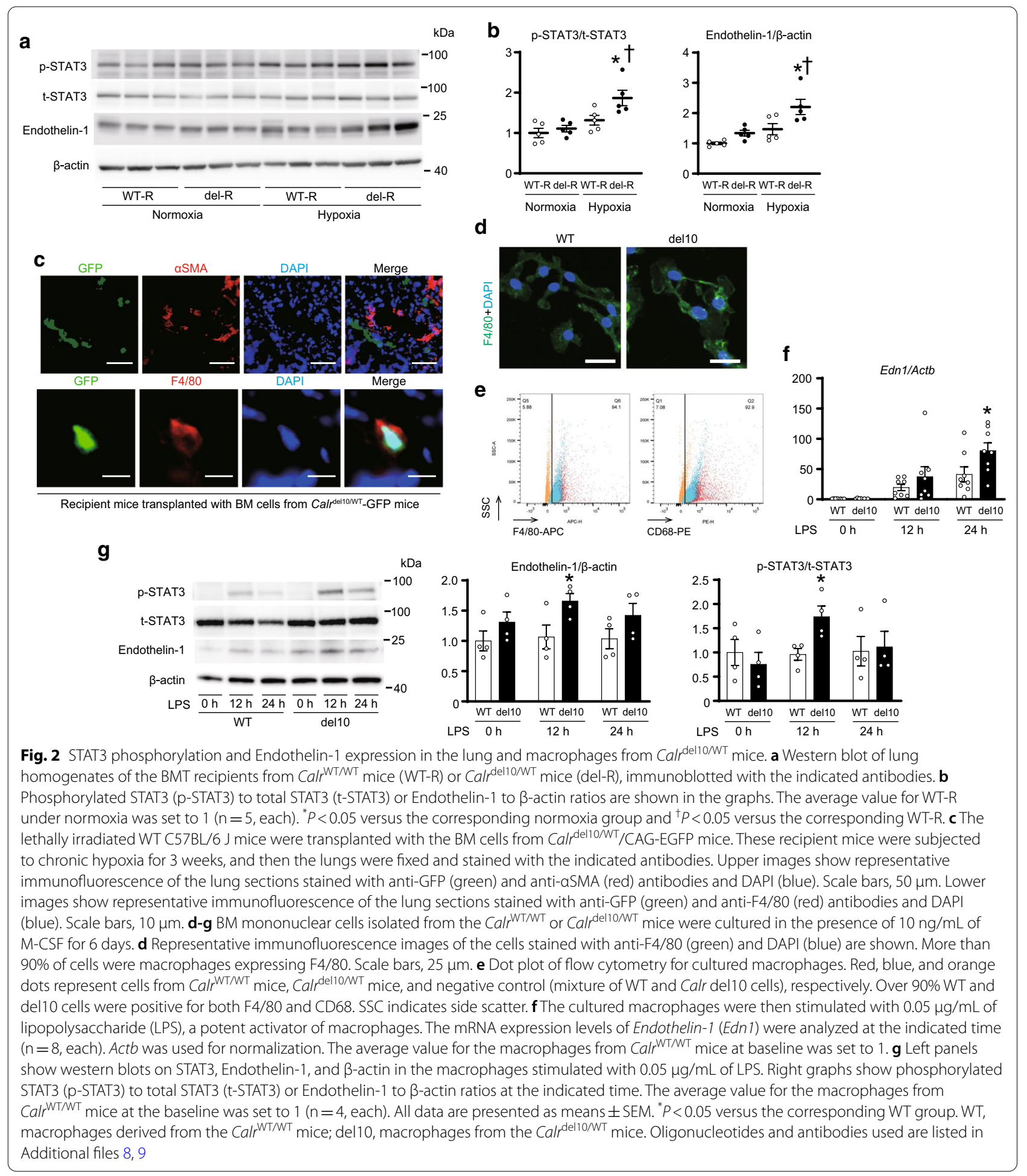

occurs with JAK-STAT activation in CALR-mutated PBMNCs similar to JAK2-mutated PB-MNCs [12]. Our murine model revealed a hematopoietic phenotype with relevance to human MPNs with CALR mutations in terms of molecular mechanisms and PH. Further study of associations between CALR mutations and $\mathrm{PH}$ or macrophage activation is needed (Additional file 10). 


\section{Abbreviations}

CALR: Calreticulin; ET: Essential thrombocythemia; MPN: Myeloproliferative neoplasm; PB: Peripheral blood; BM: Bone marrow; BMT: Bone marrow transplantation; PA: Pulmonary arterial; PH: Pulmonary hypertension; Del-R: BMT recipients from Calr ${ }^{\text {del10/WT }}$ mice; MNCs: Mononuclear cells; RV: Right ventricular; RVSP: Right ventricular systolic pressure; RV/LV + S: Right ventricle/left ventricle-plus-septum weight ratio; M-CSF: Macrophage colony-stimulating factor; WT: Wild type; WT-R: BMT recipients from Calr ${ }^{\text {WT WT }}$ mice.

\section{Supplementary Information}

The online version contains supplementary material available at https://doi. org/10.1186/s13045-021-01064-8.

\section{Additional file 1. Fig. S1: CALR proteins coded by ins 2 and del10} frameshifts in murine Calr mimicked a feature of those coded by human type 2-like CALR mutations that generated novel C termini. a Western blot of BM cells using antibody specific for the CALR N terminus (CALR-N) or C terminus (CALR-C). b Isoelectric point ( $\mathrm{pl}$ ) in human and murine CALR proteins. c Alignment of $C$ domains in mutant murine CALR from codon A352. Acidic and basic residues are in blue and red, respectively. \#: the negatively charged amino acid stretches. ${ }^{\dagger}$ : the subjects of previously reported murine CALR mutants. $\mathbf{d}$-f Identity and similarity between murine and human CALR frameshifts. The 2 MPN patients, with a mutated protein as CALR p.K375Rfs*52 (c.1124_1133del), matched the murine Calr del10 (p.K375Rfs*52 coded by Calr c.1124_1133del), although identity and similarity of the peptides were slightly different (f).

Additional file 2. Fig. S2: MPN-like phenotypes in knock-in mice with Calr frameshifts. a-b Body (a) and spleen (b) weights $(n=15-19)$. c BM nuclear cell counts $(n=4-6)$. d The proportions of BM CD71+ Ter119+ erythroblasts, $\mathrm{Gr}_{1}{ }^{+}$myeloid cells, $\mathrm{B} 220^{+} \mathrm{B}$ cells, and TCR ${ }^{+} \mathrm{T}$ cells in flow cytometry ( $n=3$ in each). e-f Histology of BM (e) and spleens ( $\mathbf{f})$. $\mathbf{g}$-h $\mathbf{h}$ The numbers of megakaryocytes per high-power field (HPF) in BM $(n=3$ in each) and spleens $(n=4-10) .\left({ }^{*} P<0.05,{ }^{* *} P<0.01\right)$.

Additional file 3. Fig. S3: Phosphorylation of STAT3 and expression of MPL, thrombopoietin receptor (TpoR). a Western blot of whole BM nuclear cells suspended in the absence of exogenous cytokines. b-c Flow cytometry gated with a lineage ${ }^{-}$fraction in BM cells. $\mathbf{b}$ Overall expression of cell-surface TpoR. Left: Histogram; right: mean fluorescence intensity (MFI). c Cell-surface expressions of TpoR and CALR. Left: heatmap plots; right: proportions of cell-surface Tpo ${ }^{+}$cells in association with CALR expression ( $n=3$ in each experiment; ${ }^{*} P<0.05$; ns: no significant difference). $\mathbf{d}$ Immunofluorescence for MPL in bone marrow. e Immunofluorescence for MPL in lung. d-e Scale bars, $50 \mu \mathrm{m}$.

Additional file 4. Fig. S4: Peripheral blood cell counts in the BMT recipients exposed to normoxia or chronic hypoxia for 3 weeks $(n=4-6)$. ( ${ }^{*} P<0.05$ versus the corresponding normoxia group).

Additional file 5. Fig. S5: Relative Edn 1 mRNA expression levels in the lung ( $n=5$, each). The average value for WT-R mice under normoxia was set to 1 . $\left({ }^{*} P<0.05\right.$ versus the corresponding normoxia group, and ${ }^{\dagger} P$ versus the corresponding WT-R mice under chronic hypoxia)

Additional file 6. Fig. S6: Gene expressions. a-e RNA sequencing (RNAseq) in LSK (lineage ${ }^{-} \mathrm{Sca}^{+}{ }^{+} \mathrm{C}-\mathrm{Kit}^{+}$) cells of an aliquot from 4 male mice of 3 months age in each sample from Calr ${ }^{\text {ins } 2 / W T}$ mice, Calr del10/WT mice, and Calr ${ }^{\text {WT/WT }}$ mice. a Principle component analysis. $\mathbf{b}$ Venn diagrams of upregulated and downregulated genes (> twofold) in LSK cells of Calr del10/ WT mice or Calr ${ }^{\text {ins } 2 / W T}$ mice relative to those of Calr ${ }^{\text {WT } / W T}$ mice. c Pathway analysis by the Ingenuity Pathway Analysis software (Qiagen). All the pathways in the comparison analysis of canonical pathways with both Z score $\geq|2|$ and $p<0.05$ in at least one of the Calrins2/WT mice and Calrdel10/ ${ }^{W T}$ mice relative to Calr ${ }^{\text {WT }}$ WT mice are shown. $\cdot$ indicates the box which did not reach the level of $Z$ score $\geq|2|$ in the genotype shown. The allow indicates Cardiac Hypertrophy Signaling pathway upregulated in both Calrins2 NT mice and Calrdel10NT mice. $\mathbf{d}$ Individual genes in the Cardiac Hypertrophy Signaling pathway. Differentially expressed genes ( $>|10|$-fold) in Calr $r^{\text {del10/WT }}$ mice relative to Calr ${ }^{\text {WT/WT }}$ mice, including EDN1 that codes Endothelin-1 (allow), are shown. e Gene set enrichment analysis (GSEA) for the JAK-STAT pathway. NES indicates normalized enrichment score; FDRq, false discovery rate $q$ value. $\mathbf{f}$-g Introduction of FLAG-Tag-inserted human WT and del52 CALR constructs into a macrophage cell line, RAW 264.7. f Western blots. g The levels of Endothelin-1 mRNA (Edn1) were analyzed in RAW 264.7 cells introduced with CALRWT or del52 after incubation under normoxia $\left(21 \% \mathrm{O}_{2}\right)$ or hypoxia $\left(10 \% \mathrm{O}_{2}\right)$ for $24 \mathrm{~h}$. Samples were taken from 3 wells for each experiment. Actb was used for normalization. The average value for cells introduced with WT CALR and incubated under normoxia was set to 1 .

Additional file 7. Table S1: Frameshifts in CALR exon 9 on the COSMIC database in hematopoietic cancers.

Additional file 8. Table S2: Oligonucleotides used in this study.

Additional file 9. Table S3: Antibodies used in this study.

Additional file 10: Supplementary methods, results, and references.

\section{Acknowledgements}

The authors would like to thank Ms. Tomiko Miura and Ms. Shoko Sato, Department of Cardiovascular Medicine, Fukushima Medical University, Fukushima, Japan, Ms. Chisato Kubo and Ms. Ayumi Haneda, Office for Gender Equality Support, Fukushima Medical University, Fukushima, Japan, and Mr. Hiroshi Nakano, Center for Molecular Genetics, Yamagata University, Yamagata, Japan, for their technical assistance.

\section{Authors' contributions}

K. Minakawa, K.U., T.Y., Y.K., T.M., K.W., Y.T., S.M., and Y.S. performed the research; O.N. generated mice; K. Mimura, K.O., T.I., and Y.T. analyzed and interpreted the data; Y.H. performed histological studies; K. Minakawa and K.E.N. interpreted the data and wrote the manuscript; K.E.N. provided editorial guidance; K.I. conceived the research, guided study design, analyzed and interpreted data, wrote the manuscript, and supervised study. All authors read and approved the final manuscript.

\section{Funding}

This work was supported by grants from JSPS KAKENHI to K.I. (15K09484, 18K08365), K.O. (15K09483) and T.Y. (19K17532), and the Uehara Memorial Foundation (201890006), and the Japanese Society of Hematology to K.I.

\section{Availability of data and materials}

The RNA sequencing data have been deposited in the Gene Expression Omnibus database (GSE152482).

\section{Declarations}

\section{Ethics approval and consent to participate}

The investigations conformed to the Guidelines for the Care and Use of Laboratory Animals published by the US National Institutes of Health (NIH publication, $8^{\text {th }}$ Edition, 2011). All efforts were addressed to minimize suffering. All studies were approved by the Animal Study Committees of Fukushima Medical University (App. No. 245, 30086) and Yamagata University (App. No. 27-153, 28-113).

\section{Consent for publication}

Not applicable.

\section{Competing interests}

T.Y.'s and K.S.'s department receives support from Janssen Pharmaceutical K.K., Japan. T.M.'s department receives support from Fukuda Denshi Co., Ltd., Japan. These companies were not associated with the contents of this study.

\section{Author details}

${ }^{1}$ Department of Blood Transfusion and Transplantation Immunology, School of Medicine, Fukushima Medical University, 1 Hikarigaoka, Fukushima 960-1295, Japan. ${ }^{2}$ Department of Cardiovascular Medicine, School of Medicine, Fukushima Medical University, Fukushima, Japan. ${ }^{3}$ Center for Molecular Genetics, Yamagata University, Yamagata, Japan. ${ }^{4}$ Department of Pulmonary Hypertension, School of Medicine, Fukushima Medical University, Fukushima, Japan. ${ }^{5}$ Department of Hematology, School of Medicine, 
Fukushima Medical University, Fukushima, Japan. ${ }^{6}$ Department of Diagnostic Pathology, School of Medicine, Fukushima Medical University, Fukushima, Japan.

Received: 23 December 2020 Accepted: 15 March 2021

Published online: 30 March 2021

\section{References}

1. Klampfl T, Gisslinger H, Harutyunyan AS, Nivarthi H, Rumi E, Milosevic $J D$, et al. Somatic mutations of calreticulin in myeloproliferative neoplasms. N Engl J Med. 2013:369:2379-90.

2. Rabinovitch M, Guignabert C, Humbert M, Nicolls MR. Inflammation and immunity in the pathogenesis of pulmonary arterial hypertension. Circ Res. 2014;115:165-75.

3. Asosingh K, Farha S, Lichtin A, Graham B, George D, Aldred M, et al. Pulmonary vascular disease in mice xenografted with human BM progenitors from patients with pulmonary arterial hypertension. Blood. 2012:120:1218-27.

4. Simonneau G, Gatzoulis MA, Adatia I, Celermajer D, Denton C, Ghofrani A, et al. Updated clinical classification of pulmonary hypertension. J Am Coll Cardiol. 2013;62:D34-41.

5. Adir Y, Elia D, Harari S. Pulmonary hypertension in patients with chronic myeloproliferative disorders. Eur Respir Rev. 2015;24:400-10.

6. Lee M-W, Ryu H, Choi Y-S, Song I-C, Lee H-J, Yun H-J, et al. Pulmonary hypertension in patients with Philadelphia-negative myeloproliferative neoplasms: a single-center retrospective analysis of 225 patients. Blood Res. 2020;55:77-84.

7. Gomez-Arroyo J, Saleem SJ, Mizuno S, Syed AA, Bogaard HJ, Abbate A, et al. A brief overview of mouse models of pulmonary arterial hypertension: problems and prospects. Am J Physiol Cell Mol Physiol. 2012:302:L977-91.
8. Tate JG, Bamford S, Jubb HC, Sondka Z, Beare DM, Bindal N, et al. COSMIC: the catalogue of somatic mutations in cancer. Nucleic Acids Res. 2019:47:D941-7.

9. Shide K. The role of driver mutations in myeloproliferative neoplasms: insights from mouse models. Int J Hematol. 2020;111:206-16.

10. Masubuchi N, Araki M, Yang Y, Hayashi E, Imai M, Edahiro Y, et al. Mutant calreticulin interacts with MPL in the secretion pathway for activation on the cell surface. Leukemia. 2020;34:499-509.

11. Ueda K, Ikeda K, Ikezoe T, Harada-Shirado K, Ogawa K, Hashimoto Y, et al. Hmga2 collaborates with JAK2V617F in the development of myeloproliferative neoplasms. Blood Adv. 2017;1:1001-15.

12. Alimam S, Villiers W, Dillon R, Simpson M, Runglall M, Smith A, et al. Patients with triple-negative, JAK2 V617F- and CALR -mutated essential thrombocythemia share a unique gene expression signature. Blood Adv. 2021;5:1059-68.

\section{Publisher's Note}

Springer Nature remains neutral with regard to jurisdictional claims in published maps and institutional affiliations.
Ready to submit your research? Choose BMC and benefit from:

- fast, convenient online submission

- thorough peer review by experienced researchers in your field

- rapid publication on acceptance

- support for research data, including large and complex data types

- gold Open Access which fosters wider collaboration and increased citations

- maximum visibility for your research: over $100 \mathrm{M}$ website views per year

At BMC, research is always in progress.

Learn more biomedcentral.com/submissions 